PII: S0038-1098(97)00371-2

\title{
PLASMON-LO-PHONON COUPLING EFFECTS ON THE DRAG RATE IN DOUBLE QUANTUM-WELL SYSTEMS
}

\author{
K. Güven and B. Tanatar \\ Department of Physics, Bilkent University, Bilkent, 06533 Ankara, Turkey
}

(Received 23 February 1997; accepted 29 April 1997 by R. Phillips)

\begin{abstract}
We study the Coulomb drag rate for electrons in a double-quantumwell structure taking into account the electron-optical phonon interactions. The full wave vector and frequency dependent random-phase approximation (RPA) at finite temperature is employed to describe the effective interlayer Coulomb interaction. The electron-electron and electron-optical phonon couplings are treated on an equal footing. The electron-phonon mediated interaction contribution is investigated for different layer separations and layer densities. We find that the drag rate at high temperatures (i.e., $T \geq 0.3 E_{F}$ ) is dominated by the coupled plasmon-phonon of the system. Including the local-field effects in an approximate way we estimate the importance of intralayer correlations to be significant. (C) 1997 Elsevier Science Ltd
\end{abstract}

\section{INTRODUCTION}

Advances in the semiconductor processing technology such as molecular-beam epitaxy and lithographic methods made it possible to manufacture high-quality quantum structures to study various physical effects. In particular, a double-quantum-well system composed of two parallel, spatially separated twodimensional (2D) electron gases is well suited to investigate the effects of mutual Coulomb interaction between the carriers in different layers. If the separation distance between the coupled quantum wells is large enough to prevent tunneling, and the quantum wells in a double-layer structure are separately contacted the so-called Coulomb drag effect is observed. The momentum and energy transfer between spatially separated electron gases were predicted to affect the transport properties of individual systems because of the Coulomb coupling [1]. The Coulomb drag effect, where a current in one layer drives a current in the other one due to the momentum loss caused by interlayer electron-electron interactions, has been observed in several experiments [2-5]. Theoretical calculations concentrated on the explanation of the observed drag rates and to formulating the framework to understand the many-body aspects of the of the drag phenomenon [6-11]. Recent measurements [12] with an applied magnetic field perpendicular to the coupled layers, are expected to inspire further work on the transport properties of systems with Landau-level quantization [13].

The temperature dependence of the observed $[2,3]$ drag rate (viz., $T_{D} \sim T^{2}$ ) identifies the Coulomb interaction as the drag mechanism. However, noticeable deviations from the $T^{2}$-behavior in the drag rate led Gramila et al. [3] to suggest that exchange of virtual phonons could be a possible mechanism, since the lowtemperature acoustic phonon mean free paths in the used samples were much larger than the layer spacing. Tso and coworkers [8] have used the momentum balance equations technique to study the effects of virtual phonon exchange on the Coulomb drag, and found that it shows a peak at low temperatures and depends weakly on the separation $d$. They obtained good agreement with experiment when a fitting parameter is used. Zhang and Takahashi [14] in their calculation of the dynamic conductivity for a doublelayer system obtained a stronger separation distance dependence. These works considered the coupling of electrons to acoustic phonons.

In this paper, we study the enhancement of the Coulomb drag rate due to the coupled plasmonphonon modes in double-quantum-well systems at zero magnetic field. Since the 2D semiconductor structures widely used in the experiments are of polar character, the electron-optical phonon interac- 
tion infuences most physical properties. We treat the electron-electron and electron-phonon interactions on an equal footing within the random-phase approximation (RPA). Our main motivation for studying the coupled plasmon-phonon effects in double-quantumwell systems comes from the recent prediction of plasmon enhanced Coulomb drag rate by Flensberg and $\mathrm{Hu}$ [9]. In contrast to the available experiments [2-5] performed at low temperatures, they found that the measured momentum transfer rate should exhibit a strong peak at temperatures around $T \sim 0.5 E_{F}$ due to the collective modes of the electronic system. This could provide a new possibility to probe the coupled plasmon modes in doublc-layer systems. The clectronacoustic phonon interactions play a crucial role in the low temperature regime. In the high-temperature experiments, the electron-optical phonon interactions are likely to be important, since they will contribute to the observed drag rate by renormalizing the bare electron-electron interaction. As these many-body effects cannot be disentangled it becomes very important to study their role for a better understanding of experimental results.

\section{MODEL AND THEORY}

We consider two infinite layers of 2D electron gases, with zero thickness, separated by a distance $d$. The separation distance is assumed to be large enough to prevent interlayer tunneling. The bare Coulomb interaction between the electrons is given by $V_{i j}(q)=$ $\left(2 \pi e^{2} / \epsilon_{\infty} q\right) e^{-q d\left(1-\delta_{i j}\right)}$ where the indices $i, j$ denote different layers. We include the high-frequency dielectric constant $\epsilon_{\infty}$, in the Coulomb interaction, as opposed to the usual $\epsilon_{0}$-approximation (static dielectric constant). The areal electron density $N$ in each layer is related to the Fermi wave vector by $N=k_{F}^{2} / 2 \pi$. We also define the dimensionless electron gas parameter $r_{s}=2^{1 / 2} /\left(k_{F} a_{B}^{*}\right)$, in which $a_{B}^{*}=\epsilon_{0} /\left(e^{2} m^{*}\right)$ is the effective Bohr radius in the semiconducting layer with background dielectric constant $\epsilon_{0}$ and electron effective mass $m^{*}$.

The Coulomb drag rate $\tau_{D}^{-1}$ between the electrons in equal density, double-quantum-wells (to lowest order in the interwire interaction) has been obtained within various theoretical approaches [6-9], to read

$$
\begin{aligned}
T_{D}^{-1}= & \frac{1}{8 \pi^{2} m^{*} N T} \\
& \times \int_{-\infty}^{\infty} d q q^{3} \int_{0}^{\infty} d \omega\left|\frac{W_{12}(q, \omega) \operatorname{Im} \chi(q, \omega)}{\sinh (\omega / 2 T)}\right|^{2}
\end{aligned}
$$

(we take $\hbar$ and $k_{B}$ equal to unity). It measures the rate of momentum transferred from one quantum-well to the other. Here, $x(q, \omega)$ is the $2 D$ dynamic susceptibility. We take $W_{12}(q, \omega)$ to be the dynamically screened effective interaction between electrons in quantumwell 1 and 2, which includes interlayer electronelectron and electron-phonon interactions on an equal footing. Thus, the total (effective) electronelectron interaction may be regarded as the sum of a Coulomb term and a contribution arising from the exchange of a virtual LO-phonon. The many-body theory for coupled electron-phonon systems [14-17] is generalized for a two-layer system interacting with bulk, dispersionless phonons with energy $\omega_{\text {LO }}$. We obtain for the effective interlayer interaction

$$
W_{12}(q, \omega)=\frac{V_{12}(q)+\psi_{12}(q, \omega)}{\varepsilon_{\mathrm{TOT}}(q, \omega)}
$$

in which

$$
\psi_{12}(q, \omega)=V_{12}(q)\left[1-\epsilon_{\infty} / \epsilon_{0}\right] \frac{\omega_{\mathrm{LO}}^{2}}{\omega^{2}-\omega_{\mathrm{LO}}^{2}+i \gamma \omega}
$$

is the LO-phonon mediated interlayer electronelectron interaction which depends on wave vector and frequency, and

$$
\begin{aligned}
\varepsilon_{\mathrm{TOT}}(q, \omega)= & {\left[1-\left(V_{11}(q)+\psi_{11}(q, \omega)\right) X(q, \omega)\right]^{2} } \\
& -\left(V_{12}(q)+\psi_{12}(q, \omega)\right)^{2} \chi^{2}(q, \omega)
\end{aligned}
$$

is the total screening function for the coupled electronLO-phonon system. In this expression, $\psi_{11}(q, \omega)$ denotes the LO-phonon mediated intralayer electronelectron interaction and we have assumed that both quantum wells have the same electron density. It is customary to write $\varepsilon_{\text {TоT }}(q, \omega)=\varepsilon(q, \omega)-P(q, \omega)$, where $\varepsilon(q, \omega)=\left[1-V_{11} X(q, \omega)\right]^{2}-V_{12}^{2} \chi^{2}(q, \omega)$ is the dielectric function for the electron system, and $P(q, \omega)$ is the correction due to the electron-phonon coupling, given by

$$
\begin{aligned}
P(q, \omega)= & 2\left(1-V_{11} \chi\right) \chi \psi_{11} \\
& +\chi^{2}\left(\psi_{12}^{2}+2 V_{12} \psi_{12}-\psi_{11}^{2}\right) .
\end{aligned}
$$

Such a decomposition in single-component systems yields dynamically screened electron-electron interaction and renormalized electron-phonon interaction. In an analogous way, we can write $[14,15,17]$

$$
W_{12}(q, \omega)=\frac{V_{12}(q)}{\varepsilon(q, \omega)}+\frac{\tilde{\varphi}(q, \omega)}{[\varepsilon(q, \omega)]^{2}},
$$

where

$$
\tilde{\Psi}_{12}(q, \omega)=\frac{\varepsilon \psi_{12}+P V_{12}}{1-P / \varepsilon}
$$


In the calculation of the drag rate $T_{D}^{-1}$, the square of the effective interaction is needed [14]

$$
\begin{aligned}
\left|W_{12}(q, \omega)\right|^{2}= & \frac{\left|V_{12}(q)\right|^{2}}{|\varepsilon(q, \omega)|^{2}}+\frac{2 V_{12}(q)}{|\varepsilon(q, \omega)|^{2}} \operatorname{Re}\left\{\frac{\tilde{\psi}_{12}}{\varepsilon(q, \omega)}\right\} \\
& +\frac{\left|\tilde{\psi}_{12}(q, \omega)\right|^{2}}{|\varepsilon(q, \omega)|^{4}}
\end{aligned}
$$

The electron-phonon interaction contribution to the drag rate is determined by the second and the third terms in equation(7). Tso et al. [8] neglected the real phonon-exchange (third term) in their study of electron-acoustic phonon coupling and showed that the virtual phonon process (second term) can explain the observed low temperature behavior of $T_{D}^{-1}$ in double-quantum-well systems. Zhang and Takahashi [14] argued that the real phonon term is equally important. In this work we employ equation (2) without making any other approximations and calculate $T_{D}^{-1}$ for the coupled electron-phonon and uncoupled electron systems. By uncoupled electron system we mean the Coulomb coupled double-layer electron system without the phonon terms in equation (7).

\section{RESULTS AND DISCUSSION}

We use the material parameters appropriate for a GaAs system for which the recent experiments [3-5] on drag rate are performed. The high-frequency and static dielectric constants are given, respectively, by $\epsilon_{\infty}=10.9$, and $\epsilon_{0}=12.9$. The LO-phonon energy which we take to be dispersionless is $\omega_{\mathrm{LO}}=36.8 \mathrm{meV}$.

We evaluate the Coulomb drag rate $\tau_{D}^{-1}$ using the effective interaction obtained for a double-layer GaAs system. We retain the full wave vector, frequency, and temperature dependence in $\varepsilon_{\text {TOT }}(q, \omega)$. Figure 1 shows the temperature dependence of the drag rate scaled by $T^{2}$ for a double-layer system with $d=4 a_{B}^{*}$ and $r_{s}=1$. Solid and dashed lines are for the coupled and uncoupled systems, respectively. We observe that electron-phonon coupling has negligible effect up to $T \sim 0.3 E_{F}$, after which collective modes dominate the momentum transfer rate. The enhancement in $T_{D}^{-1} / T^{2}$ due to plasmons for high temperatures is somewhat reduced when coupled plasmon-phonon modes are present. Such a reduction in the scattering rates when electron-phonon coupling is considered has also been obtained in other many-body calculations [15]. The dotted line in Fig. 1 is the result for uncoupled system within the $\epsilon_{0}$-approximation, where the bare Coulomb interaction reads $V_{i j}(q)=\left(2 \pi e^{2} / \epsilon_{0} q\right) e^{-q d\left(1-\delta_{i j}\right)}$. The collective excitation modes are obtained from the solution of $\varepsilon_{\mathrm{TOT}}\left(q, \omega_{\mathrm{pl}}(q)\right)=0$. The uncoupled double-

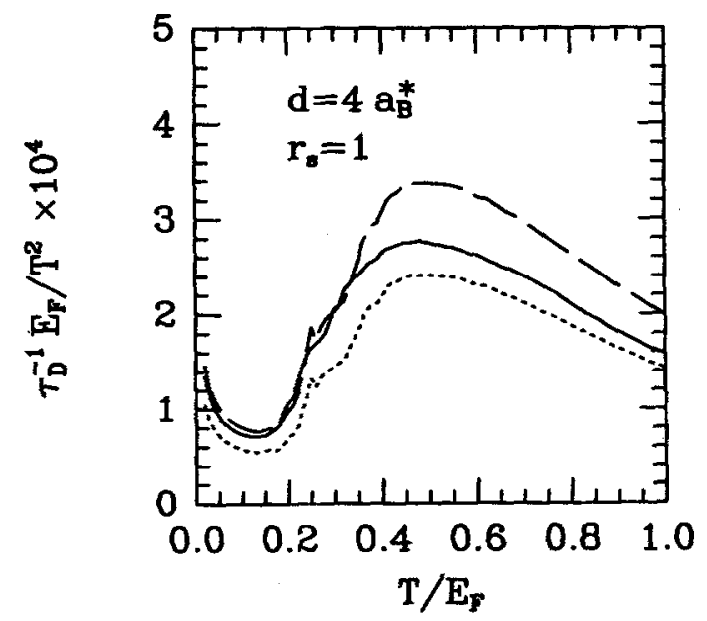

Fig. 1. The scaled drag rate $T_{D}^{-1} / T^{2}$ as a function of temperature for a double-quantum-well system with $d=4 a_{B}^{*}$, and $r_{s}=1$. The solid and dashed lines are for coupled and uncoupled systems, respectively, whereas the the dotted line shows the uncoupled systems within the $\epsilon_{0}$-approximation.

layer electron system has optical and acoustic plasmon modes. Coupling to LO-phonons, modifies the plasmon and phonon dispersions, so that the coupled LOphonon mode slightly increases and coupled plasmon modes slightly decrease.

It has been shown $[3,7,8]$ that for low temperatures $\left(T \lesssim 0.2 E_{F}\right)$, when plasmon enhancement is negligible, the drag rate behaves as $\tau_{D}^{-1} \sim d^{-4}$. This is mainly due to the cut-off in the interlayer Coulomb interaction and static screening effects operative at small frequencies [9] $\left(\omega<<q v_{F}\right.$ where $v_{F}$ is the Fermi velocity). In the plasmon dominated regime Flensberg and $\mathrm{Hu}$ [9] found that $T_{D}^{-1}$ approximately has $d^{-3}$ dependence. In Fig. 2, we show $\tau_{D}^{-1} d^{4} / T^{2}$ for different layer separations, for the coupled electron-phonon and uncoupled electron systems. At low $T$, both the coupled and uncoupled drag rates coincide, reflecting the $d^{-4}$ scaling. However, at high temperatures, in the region where collective modes become important, the size of the peak increases with increasing $d$, which indicates a slower fall off than $d^{-4}$. We plot in Fig. 3 the dependence of $T_{\max }$ on layer separation $d$, which shows that the peak position for the coupled system moves slightly to lower temperatures.

It is known that the RPA becomes less reliable for electron densities such that $r_{s}>1$ (low density). For double-layer electron-hole systems it was found necessary to go beyond the RPA to obtain reasonable agreement with the observed drag rates [10]. Correlation effects are also shown to be important for electronelectron systems in the calculation of transresistiv- 


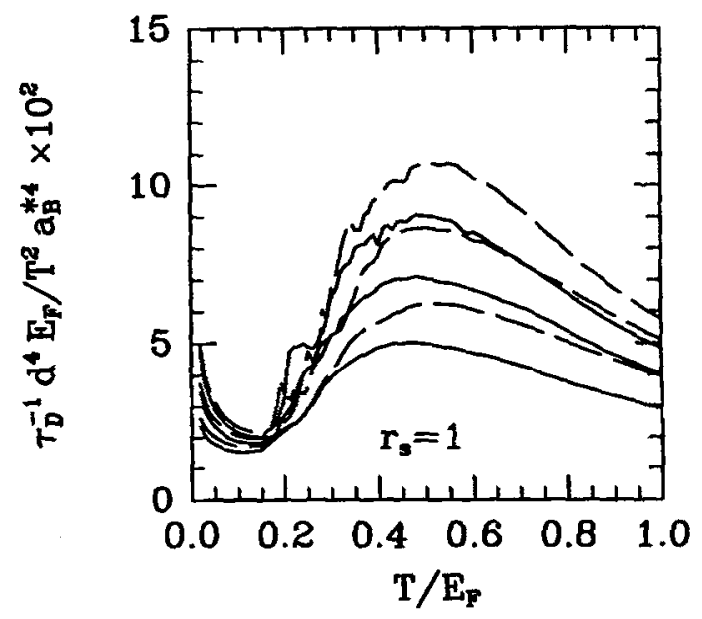

Fig. 2. The scaled drag rate $T_{D}^{-1} d^{4} / T^{2}$ as a function of temperature at $r_{s}=1$ for different well separations $d$. The curves from bottom to top indicate $d=3 a_{B}^{*}, 4 a_{B}^{*}$, and $5 a_{B}^{*}$, respectively. The solid and dashed lines are for coupled electron-phonon and uncoupled electron systems, respectively.

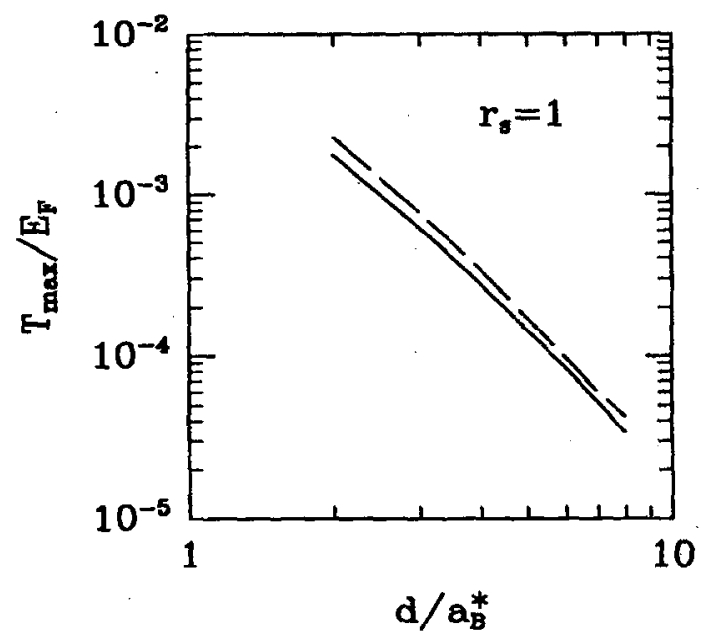

Fig. 3. The dependence of $T_{\max }$ on layer separation for coupled (solid) and uncoupled (dashed) systems, at $r_{s}=1$.

ity [18]. We incorporate the correlation effects in an approximate way using local-field corrections. A simplified attempt to go beyond the RPA is provided by the Hubbard approximation in which the Pauli hole around electrons is taken into account. Neglecting the interlayer correlations but including the intrawire exchange effects (i.e., Hubbard approximation) we take $[10,18] G_{i j}(q)=q \delta_{i j} / 2\left(q^{2}+k_{F}^{2}\right)^{1 / 2}$, so that the bare Coulomb interactions are replaced by $V_{i j}(q) \rightarrow$ $V_{i j}(q)\left[1-G_{i j}(q)\right]$ in the screening function $\varepsilon_{\text {TOT }}(q, \omega)$. The interlayer local-field correction should decrease with increasing separation $d$, thus our simple approx-

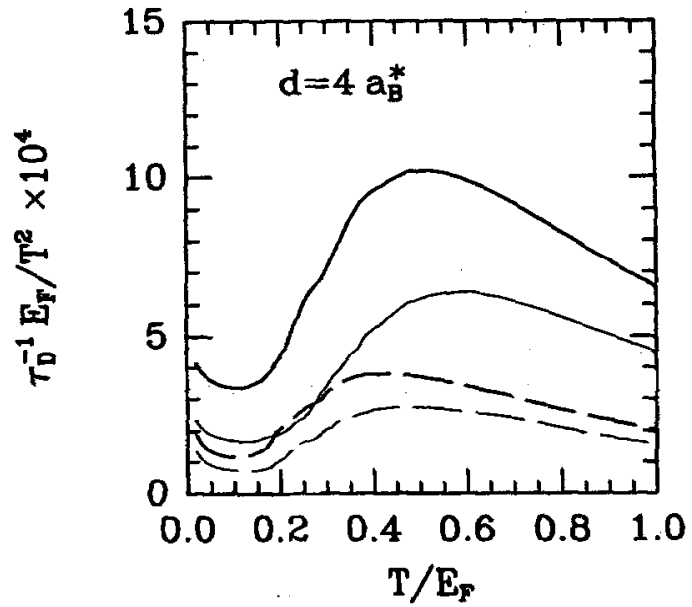

Fig. 4. The drag rate for the coupled system at $d=$ $4 a_{B}^{*}$ and $r_{s}=1$ (dashed lines) and $r_{s}=1.5$ (solid lines), with (thick lines) and without (thin lines) the local-field effects.

imation is justified. A calculation by Świerkowski et $a l$. [18] shows that the $G_{12}$ affects the transresistivity in double-layer electron systems very little. However, the short-range correlations built in via the selfconsistent scheme yield a substantial increase. Even the approximate approach of using the Hubbard localfield corrections gives noticeably different results than the RPA. In Fig. 4, we show the drag rate with (thick lines) and without (thin lines, RPA) the local-field corrections for $r_{s}=1$ (dashed) and $r_{s}=1.5$ (solid). In general, the correlation effects increase the calculated drag rate. The peak structure in $\tau_{D}{ }^{1} / T^{2}$ due to plasmon enhancement shifts slightly to lower temperatures. It would be interesting to develop more accurate localfield corrections taking their temperature dependence into account [19]. The dependence of the drag rate on electron density parameter $r_{s}$ in each layer is also seen in Fig. 4. As the density is decreased, the exchangecorrelation effects become more appreciable and $T_{D}^{-1}$ is enhanced. The plasmon peak shifts to higher temperatures indicating once again that collective modes are responsible for the observed behavior. We note that the coupled plasmon-phonon mode effects start to deviate from the uncoupled system results at a higher temperature for lower density system.

In summary, we have considered the Coulomb drag effect between two parallel quantum-wells. The temperature dependence of the drag rate is significantly enhanced when a dynamically screened effective interlayer interaction is used. This enhancement is due to the coupled plasmon-LO-phonon modes the doublewell system. So far, the experiments [2-5] measuring the Coulomb drag rate in double-layer systems were 
carried out at low temperatures $\left(T<<E_{F}\right)$. To observe the plasmon effects suggested by Flensherg and Hu [9] the region of high temperatures $T \sim E_{F}$ has to be probed. In the high temperature experiments it will also be possible to observe the coupled plasmonphonon effects discussed here. The local-field effects describing correlations beyond the simple RPA seem to be quite important for low densities affecting the drag rate considerably.

Note added in proof-We have recently became aware of new high temperature drag measurements. [20] Our calculations including the LO-phonon coupling effects seem to be in the right direction to achieve better agreement with the experimental results.

Acknowledgements-This work is partially supported by the Scientific and Technical Research Council of Turkey (TUBITAK) under grant no TBAG-AY/123. We thank Dr. M. Z. Gedik and Professor C. M. Sotomayor-Torres for useful discussions.

\section{REFERENCES}

1. Price, P. J., Physica B, 117, 1983, 750; Pogrebinskii, M. B., Sov. Phys. Semicond, 11, 1977, 372; Price, P. J., in The Physics of Submicron Semiconductor Devices (Edited H. L. Grubin, D. K. Ferry and C. Jacoboni). Plenum, New York, 1988.

2. Solomon, P. M., Pricc, P. J., Frank, D. J. and La Tulipe, D. C., Phys. Rev. Lett., 63, 1989, 2508.

3. Gramila, T. J., Eisenstein, J. P., MacDonald, A. H., Pfeiffer, L. N. and West, K. W., Phys. Rev. Lett., 66, 1991, 1216; Phys. Rev. B, 47, 1993, 12,957; Physica $B, 197,1994,442$.

4. Sivan, U., Solomon, P. M. and Shtrikman, H., Phys. Rev. Lett., 68, 1992, 1196.

5. Rubel, H., Linfield, E. H., Ritchie, D. A., Brown, K. M., Pepper, M. and Jones, G. A. C., Semicond. Sci. Tech., 10, 1995, 1229.

6. Laikhtman, B. and Solomon, P. M., Phys. Rev. B,
41, 1990, 9921; Boiko, I. I. and Sirenko, Yu. M., Phys. Status Solidi (b), 159, 1990, 805.

7. Zheng, L. and MacDonald, A. H., Phys. Rev. B, 48, 1993, 8203; Jauho, A.-P. and Smith, H., Phys. Rev. $B, 47,1993,4420$.

8. Tso, H. C., Vasilopoulos, P. and Peeters, F. M., Phys. Rev. Lett., 68, 1992, 2516; ibid, 70, 1993, 2146; Vasilopoulos, P. and Tso, H. C., in Condensed Matter Theories, Vol. 8, p. 81 (Edited by L. Blum and F. B. Malik). Plenum, New York, 1993.

9. Flensberg, K. and Hu, B. Y.-K., Phys. Rev. Lett., 73, 1994, 3572; Phys. Rev. B, 52, 1995, 14,796.

10. Świerkowski, L., Szymański, J. and Gortel, Z. W., Phys. Rev. Lett., 74, 1995, 3245.

11. Kamenev, A. and Oreg, Y., Phys. Rev. B, 52, 1995, 7516; Flensberg, K., Hu, B. Y.-K., Jauho, A.-P. and Kinaret, J., Phys. Rev. B, 52, 1995, 3245.

12. Hill, N. P. R., Nicholls, J. T., Linfield, E. H., Pepper, M., Ritchie, D. A., Hamilton, A. R. and Jones, G. A. C., J. Phys. Condens. Matter, 8, 1996, L557.

13. Bønsager, M.C., Flensberg, K., Hu, B.-Y.K. and Jauho, A.-P., -it Phys. Rev. Lett., 77, 1996, 1366; Wu, M.W., Cui, H.L. and Horing, N.J.M., Mod. Phys. Lett. B, 10, 1996, 279; Tso, H.C. and Vasilopoulos, P., Phys. Rev. B, 45, 1992, 1333.

14. Zhang, C. and Takahashi, Y., J. Phys. Condens. Matter, 5, 1993, 5009 (1993).

15. Jalabert, R. and Das Sarma, S., Phys. Rev. B, 40, 1989, 9723; Das Sarma, S. and Mason, B. A., Ann. Phys., 163, 1985, 78; Das Sarma, S., Jain, J. K. and Jalabert, R., Phys. Rev. B, 37, 1988, 6290.

16. Tzoar, N. and Zhang, C., Phys. Rev. B, 35, 1987, 7596.

17. Sanborn, B. A., Phys. Rev. B, 51, 1995, 14,256.

18. Świerkowski, L., Szymański, J. and Gortel, Z. W., Sur. Sci., 361/362, 1996, 130.

19. Tanaka, S. and Ichimaru, S., J. Phys. Soc. Jpn, 55, 1986, 2278; Schweng, H., Böhm, H. M., Schinner, A. and Macke, W., Phys. Rev. B, 44, 1991, 13,291.

20. Hill, N.P.R., Nicholls, J.T., Linfield, E.H., Pepper, M., Ritchie, D.A., Jones, G.A.C., Hu, B.Y.-K. and Flensberg, K., Phys. Rev. Lett., 78, 1997, 2204. 